\title{
Rapid quantitative analysis of six flavonoids in licorice by ultra-performance convergence chromatography
}

\author{
Li LI' ${ }^{1}$, Wenyi ZHU ${ }^{1}$, Jingxiu YANG ${ }^{1}$, Xiaolan $\mathrm{LIU}^{1}$, Yinmao DONG ${ }^{1 *}$ (1)
}

\begin{abstract}
Objective: This study was to establish a rapid quantitative method for determination of six characteristic flavonoids, including licochalcone A (1), liquiritigenin (2), glabridin (3), isoliquiritigenin (4), liquiritoside (5), and isoliquiritoside (6) in licorice by a ultra-performance convergence chromatography ( $\left.\mathrm{UPC}^{2}\right)$ apparatus equipped with a photodiode-array detector (PDA). Methods: The flavonoids studied were separated on an ACQUITY UPC ${ }^{2}$ TM Torus 2-PIC column $(2.1 \times 100 \mathrm{~mm}, 1.7 \mu \mathrm{m})$ using gradient elution (85:15-70:30) with a mobile phase consisting of $\mathrm{CO}_{2}$ and methanol: formic acid (99.8:0.2, v/v), back pressure of 2,000 psi, flow rate of $0.8 \mathrm{~mL} / \mathrm{min}$, and detection at $254 \mathrm{~nm}$. This method was further applied for the analysis of the flavonoids present in 10 batches of licorice roots samples. Results: Good linearity $\left(\mathrm{R}^{2}=0.9990-0.9998\right)$ was achieved and limits of detection (LOD) and quantification (LOQ) ranging from 0.12 to $0.49 \mu \mathrm{g} / \mathrm{mL}$ and 0.46 to $1.61 \mu \mathrm{g} / \mathrm{mL}$, respectively. The relative standard deviations (RSD) for repeatability and reproducibility were $2.30-2.80 \%$ and $2.30-2.98 \%$, respectively. The flavonoids distributed differently in Glycyrrhiza uralensis, Glycyrrhiza glabra, and Glycyrrhiza inflate. Conclusion: The established rapid quantitative analysis method was environment friendly and suitable to the quality control of species licorice.
\end{abstract}

Keywords: licorice; UPC²/PDA; quantify; flavonoids.

Practical Application: The environment friendly method was suitable to the quality control of licorice.

\section{Introduction}

The Glycyrrhiza genus consists of about 30 species and is widely distributed all over the world. In China and Europe, the roots and rhizomes of Glycyrrhiza uralensis, Glycyrrhiza glabra, and Glycyrrhiza inflata are used as licorice without discrimination, whereas only the first two species are used in the United States and Japan (Commission Chinese, 2015; Ministry of Health, Labour and Welfare, 2011).

Licorice is a tonic herbal medicine which is included extensively as a component herb in approximately $60 \%$ of all traditional Chinese medicine (TCM) prescriptions because of the synergistic therapeutic effects it produces when combined with other herbal medicines (Wang \& Yang, 2007). In previous studies, significant differences were found in the secondary metabolite profiles of the three species (G. uralensis, G. glabra, and G. inflata), especially concerning their content of free phenolic compounds, including coumarins and flavonoids (Song et al., 2017). Importantly, flavonoids in licorice including chalcones, isoflavones, flavonones, flavanonols, isoflavenes, and arylcoumarins (Lee et al., 2007) have drawn considerable interest due to their pharmacological properties, especially those related to skin health improvement, such as skin-whitening, sun-screening, anti-inflammatory, and anti- oxidative activities (Simmler et al., 2013; Yokota et al., 1998). Currently, the quality control method of licorice was mainly established on the high performance liquid chromatography (HPLC) and liquid chromatograph-mass spectrometer (LC/MS) (Yang et al., 2016; Zhang, 2016). As a chromatography technology used in the instruments developed by Waters Corporation (Milford, MA, USA), ultra-performance convergence chromatography (UPC ${ }^{2}$ integrates the benefits of the supercritical fluid chromatography (SFC) technology with the performance of UPLC. UPC ${ }^{2}$ is a standalone, viable technique that is cost effective, sustainable, and uses green technology that lowers the use of organic solvents. This method is recognized as a substituent of the normal-phase, the orthogonality, and complementary to reversed-phase HPLC (Gong et al., 2014; Qi et al., 2016; Zhou et al., 2014).

In the present study, a new UPC $2 / P D A$ method for simultaneous separation and determination of six flavonoids was established and developed to efficiently validate and quantify the flavonoids in the collected licorice root samples from different producing areas of China. The shorter retention time, improved analytical sensitivity, economic experimental cost, and environment friendly solvent was the advantage of the new establish methods.

\section{Chemicals and reagents}

HPLC-grade methanol was purchased from Merck (Germany). Ultra-pure water $(18.2 \mathrm{M} \Omega$ ) was prepared with a Milli-Q water purification system (Millipore, Bedford, MA, USA). Licochalcone A (1), liquiritigenin (2), glabridin (3), isoliquiritigenin (4), liquiritoside (5), and isoliquiritoside (6) were purchased from 
Beijing Simianti Tech. Co., Ltd. (Beijing, China) and used as reference standards (Table 1). The purity of all the reference compounds was greater than $98 \%$ as determined by HPLC analysis. All other reagents were of analytical grade. Carbon dioxide (99.99\%) was purchased from Beijing Reiz Hanxing Technology Co. Ltd. (Beijing, China).

\section{Plant materials and sample preparation}

10 batches of licorice were collected from the different areas of China and were identified by Dr. Lijia Xu, a professor in the Institute of Medicinal Plant Development (IMPLAD), affiliated with the Chinese Academy of Medical Sciences (CAMS).

Table 1. The structures of licochalcone A, liquiritigenin, glabridin, isoliquiritigenin, liquiritoside, isoliquiritoside.

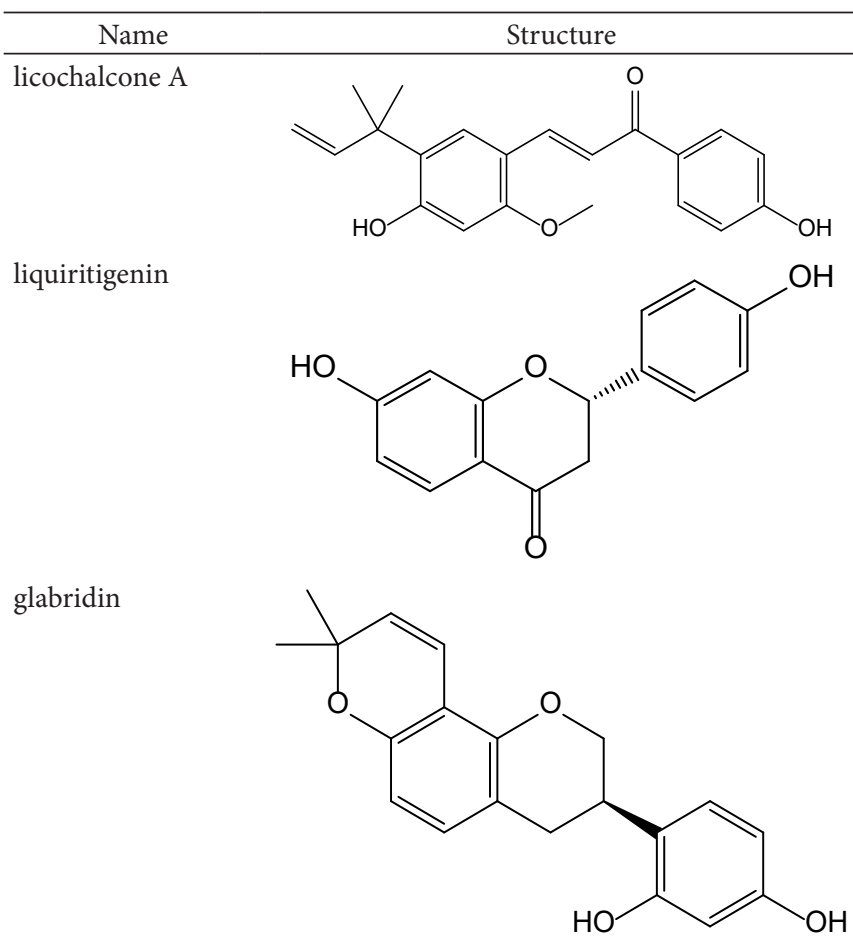

isoliquiritigenin<smiles>O=C(/C=C/c1ccc(O)cc1)c1ccc(O)cc1O</smiles>

liquiritoside<smiles>O=C1CC(c2ccc(OC3OC(CO)[C@@H](O)[C@@H](O)[C@H]3O)cc2)Oc2cc(O)ccc21</smiles>

isoliquiritoside

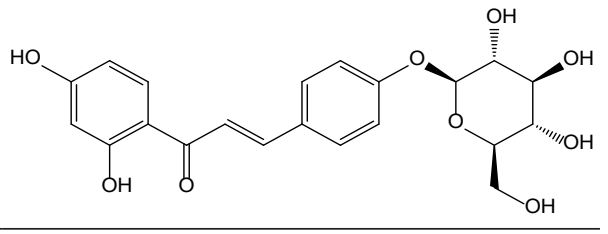

Air-dried samples $(3.0 \mathrm{~g})$ were sieved and subjected to ultrasonic extraction with $50 \mathrm{~mL}$ of $95 \%$ ethanol $(v / v)$ for $30 \mathrm{~min}$. The filtered extraction solutions of 3 repetitions were combined, concentrated, and freeze-dried. Then, the dry powder of the extract was dissolved to $5.0 \mathrm{mg} / \mathrm{mL}$ with methanol. The extracts were filtered through a $0.22-\mu \mathrm{m}$ filter, and $3.0 \mu \mathrm{L}$ of each of them was injected into the UPC 2 system for analysis.

\section{Standards and quantification}

Each solution $(1.0 \mathrm{mg} / \mathrm{mL})$ of licochalcone A (1), liquiritigenin (2), glabridin (3), isoliquiritigenin (4), liquiritoside (5), and isoliquiritoside (6) were prepared. Then, each of them was further dissolved into a concentration of $0.167 \mathrm{mg} / \mathrm{mL}$ for the mixed standard solution. The solution was stored in dark glass bottles at $4^{\circ} \mathrm{C}$. The working standard solutions were freshly prepared by diluting suitable amounts of the above solutions with methanol before injection. A linear relationship between peak areas and concentrations was observed, and the linear regression model was applied to determine the contents of flavonoids in the selected samples.

\section{Apparatus and separation condition}

Chromatography was performed using an ACQUITY UPC $^{2}$ system (Waters, Milford, MA, USA) with binary solvent manager, sample manager, column manager, and convergence chromatography manager. Quantitative analysis was performed on an ACQUITY UPC2TM Torus 2-PIC column $(2.1 \times 100 \mathrm{~mm}$, $1.7 \mu \mathrm{m}$; Waters, MA, USA) maintained at $40{ }^{\circ} \mathrm{C}$ using a mobile phase containing $\mathrm{SCCO}_{2}$ (purity $\geq 99.99 \%, \mathrm{~A}$ ) and methanol $(0.2 \%$ formic acid, B). The gradient elution of $A$ and $B$ was performed as follows: $15-20 \% \mathrm{~B}$ at $0-2 \mathrm{~min}, 20-25 \% \mathrm{~B}$ at $2-4 \mathrm{~min}, 25-30 \% \mathrm{~B}$ at $4-6 \mathrm{~min}, 30-35 \% \mathrm{~B}$ at $6-8 \mathrm{~min}$. The pressure was maintained at 2,000 psi by an auto-back pressure regulator (ABPR). A flow rate of $0.8 \mathrm{~mL} / \mathrm{min}$ was used in our study. The auto-sample temperature was set at $25^{\circ} \mathrm{C}$. The wavelength range of the PDA detector was $190-400 \mathrm{~nm}$, and the detection wavelength was set at $254 \mathrm{~nm}$ for analysis after the comprehensive consideration of the maximum absorption of the six standards. An injection volume of $3.0 \mu \mathrm{L}$ was used for each of the samples. Data were processed using Empower 3 software (Waters Corporation, Milford, MA, USA).

\section{$6 \mathrm{UPC}^{2}$ method validation}

Our newly developed $\mathrm{UPC}^{2}$ method was validated in terms of linearity, precision, and accuracy according to the $\mathrm{ICH}$ guidelines (ICH Expert Working Group, 1996; Li et al., 2012). A calibration curve was constructed by running the mixed standards of different concentrations in triplicate. The correlation coefficient was determined using a linear regression model. By injecting a series of dilute solutions with known concentrations, the limit of detection (LOD) and the limit of quantification (LOQ) for the standard compounds were estimated to have signal-to-noise ratios $(\mathrm{S} / \mathrm{N})$ of 3 and 10 , respectively.

\section{Analysis of licorice root samples}

The newly established $\mathrm{UPC}^{2} / \mathrm{PDA}$ method was applied for simultaneous determination of the six flavonoids in 10 batches of licorice root samples. The contents were calculated using the 
standard curves. Duncan's multiple-range test using the Statistical Package for Social Studies (SPSS) (IBM Corp., Armonk, NY, USA) were used to analyze the results.

\section{Results and discussion}

\subsection{Optimization of extraction conditions}

Different extraction solvents were employed: $50 \%(v / v)$ ethanol, 75\% $(v / v)$ ethanol, ethanol, 50\% $(v / v)$ methanol, $75 \%$ $(v / v)$ methanol and methanol. Finally, we selected ethanol as a solvent due to the highest values of the target compounds reached in the peak areas, and the least effect of the impurities. Other experimental factors, including extraction repetitions (1-3 times) and extraction times $(15,25,30$, or $40 \mathrm{~min})$, were also tested and evaluated. As a result, the optimized extraction method was suggested to be threefold ultrasonication with ethanol for $30 \mathrm{~min}$.

\subsection{Optimization of chromatographic conditions}

The efficiency of UPC ${ }^{2}$ separation of all six flavonoids was investigated by tests based on different chromatographic conditions, including various columns, mobile phases, gradient elution, back pressures, flow rates, and column temperatures (Qi et al., 2016). To obtain a high-resolution data within a reasonable time, four different types of columns were evaluated, including ACQUITY UPC ${ }^{2}$ TM Torus 1 -AA $(2.1 \times 100 \mathrm{~mm}$, $1.7 \mu \mathrm{m})$, ACQUITY UPC ${ }^{2} \mathrm{TM}$ BEH $(3.0 \times 100 \mathrm{~mm}, 1.7 \mu \mathrm{m})$, ACQUITY UPC 2 TM Torus 2- PIC $(2.1 \times 100 \mathrm{~mm}, 1.7 \mu \mathrm{m})$, and ACQUITY UPC 2 TM Torus Diol $(2.1 \times 100 \mathrm{~mm}, 1.7 \mu \mathrm{m}$, Waters, MA, USA). A better peak shape and resolution were obtained when Torus 2-PIC column was used.
To improve the separation of the six flavonoids investigated, different co-solvent systems were tested, including methanol, acetonitrile, methanol: acetonitrile (1:1), methanol: formic acid (99.8:0.2, $v / v)$, and methanol: acetic acid (99.8:0.2, v/v). Optimal results were obtained when solvent $\mathrm{B}$, consisting of methanol: formic acid (99.8:0.2, $v / v)$, and a gradient elution mode were used. The change in the density of the supercritical $\mathrm{CO}_{2} \mathrm{via}$ back pressure directly affected the eluting power of the whole supercritical fluid system.

In the present study, an optimal back pressure of 2, $000 \mathrm{psi}$ was applied in the UPC ${ }^{2}$ analysis conducted. The most suitable column temperature and flow rate were $40{ }^{\circ} \mathrm{C}$ and $0.8 \mathrm{~mL} / \mathrm{min}$, respectively. The determination of flavonoids in the licorice root samples was accomplished using the optimized chromatographic conditions. Each analyte was identified by comparison between the retention times of the peaks of the standard reference compounds and those of the selected samples obtained by UPC 2 under the same conditions.

\subsection{Method validation}

As can be seen in Figures 1 and 2, the newly established $\mathrm{UPC}^{2}$ method offers adequate separation selectivity among the six flavonoids, and the unidentified peaks in the selected samples did not interfere with the analytes. For identification of flavonoids, the retention times and spectra were used. The linearity was calculated based on the injection of five increasing concentrations of the standard solution. The linear regression analysis showed good linearity for each compound $\left(R^{2}>0.9990\right)$ (Table 2$)$, which allowed the acquisition of reliable and effective analytical data for the licorice root samples studied. The LODs ( $/ \mathrm{n}=3)$ and LOQs $(\mathrm{s} / \mathrm{n}=10)$ are listed in Table 2 . The RSD of the reproducibility and repeatability were $2.30-2.84 \%$ and $2.30-2.98 \%$ (Table 2 ),

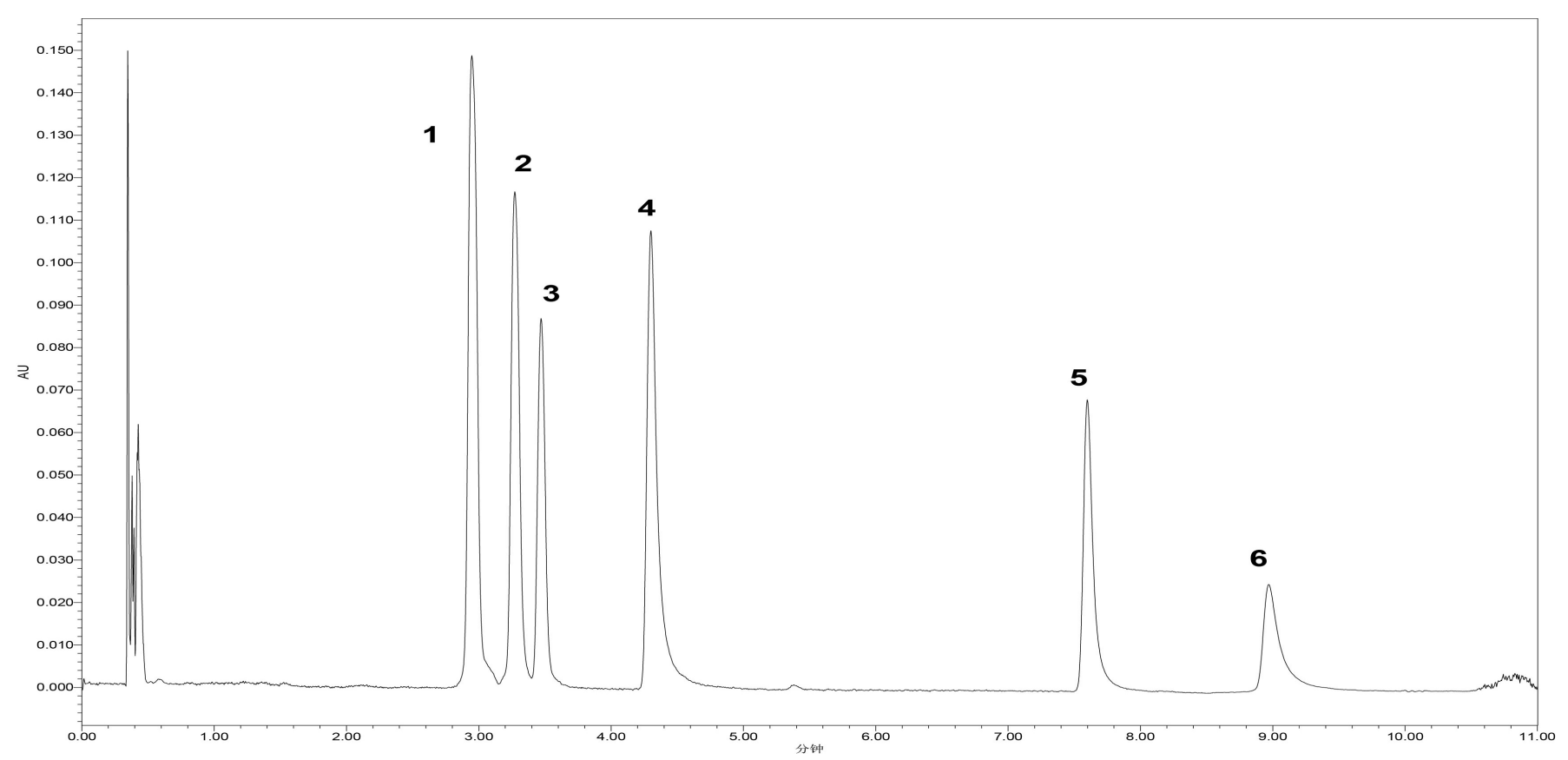

Figure 1. Typical chromatogram of standard mixture of six flavonoids. Peaks numbering: licochalcone A (1), liquiritigenin (2), glabridin (3), isoliquiritigenin (4), liquiritoside (5), isoliquiritoside (6). 


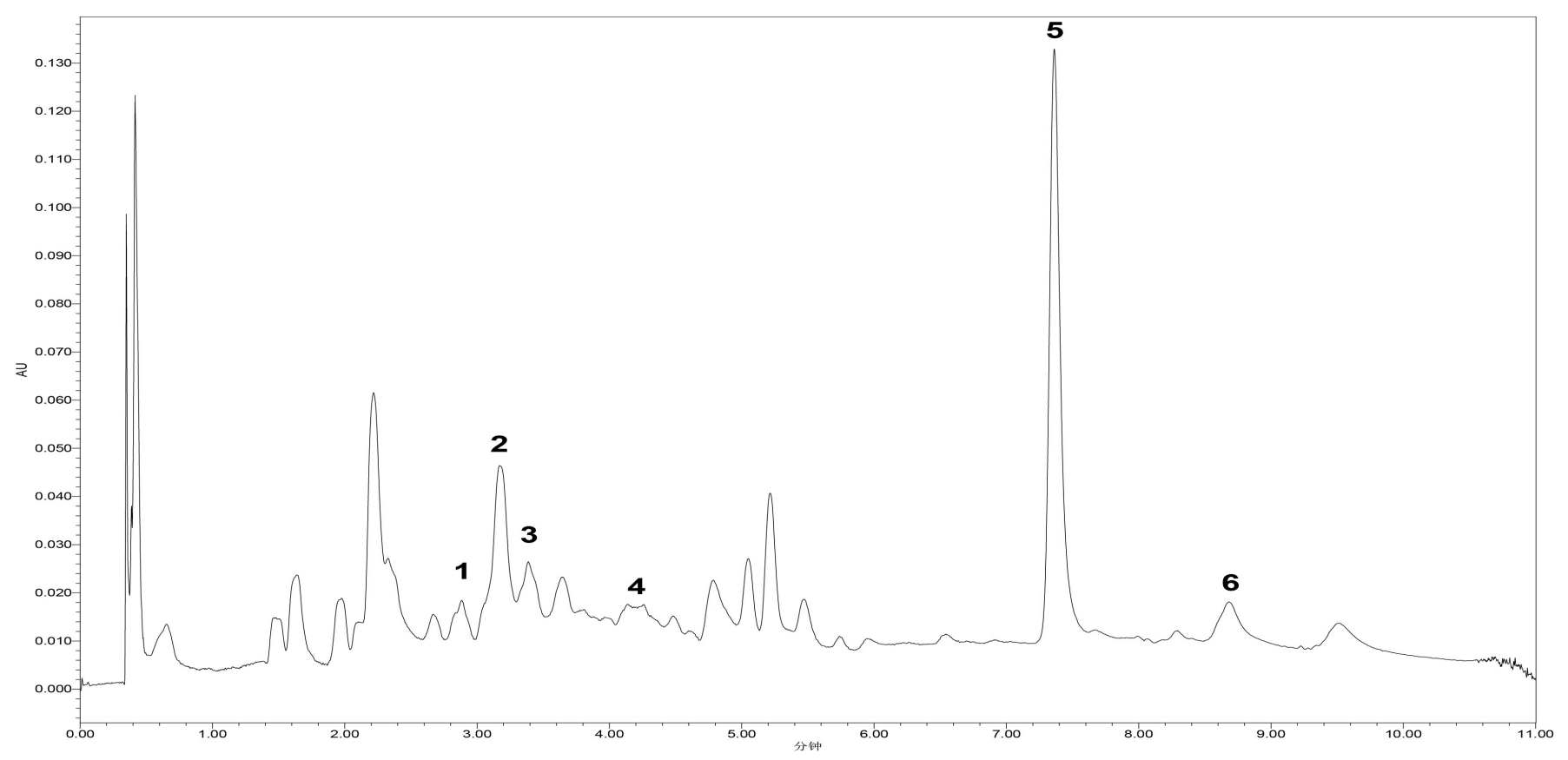

Figure 2. Typical chromatogram of licorice root sample (batch 5 from Province Yunnan). the numbers as in Fig. 1.

Table 2. Linear regression, LOD, LOQ, and precision for the determination of six flavonoids by the $\mathrm{UPC}^{2}$ methods.

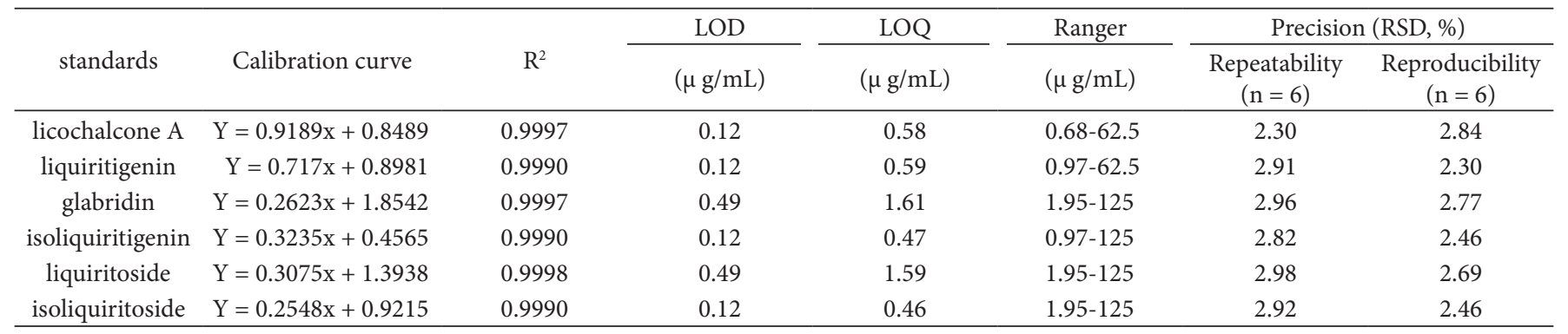

Calibration curve: $\mathrm{y}=\mathrm{mx}+\mathrm{b}$, where $\mathrm{y}$ is the integrated peak area and $\mathrm{x}$ is the concentration in $\mu \mathrm{g} / \mathrm{mL}$.

respectively, indicating that the precision of this $\mathrm{UPC}^{2}$ method is good. Therefore, we can conclude that the overall analytical $\mathrm{UPC}^{2}$ method is fast, accurate, and suitable for the quantitative analysis of many samples.

\subsection{Analysis of flavones in selected licorice root samples}

In Chinese pharmacopoeia, the roots and rhizomes of G. uralensis, G. glabra, and G. inflata are used as licorice without discrimination. The main producing areas have been reported to change from Province Shanxi to the northwest of China (Provinces Ningxia, Neimenggu, Gansu, and Xinjiang) in recent years (Gao et al., 2017). Hybridization could have resulted from the overlapped cultivation of different Glycyrrhiza species and insufficient isolation among species (Chen et al., 2017). G. uralensis is the most widely used species that constitutes more than $90 \%$ of the total licorice production. Three species licorice classified by using statistical methods one-way analysis of variance (ANOVA) followed by Duncan's multiple-range test using the Statistical Package for Social Studies (SPSS) (IBM Corp., Armonk, NY, USA).
In the present paper, 10 batches obtained from different markets in China were analyzed by the newly established method. The results $(\mu \mathrm{g} / \mathrm{g})$ are shown in Table 3. All data are presented as mean \pm standard deviation (SD). The contents of the tested six flavonoids were considerably different. For example, $G$. inflata (batches 1 and 10) contained remarkably more abundant 2'-H chalcones (e.g., licochalcones A), whereas G. glabra (batch 4) contained more abundant 8-cyclized isoprenyl isoflavanes (glabridin) than the other two species, which was consistent with previously reported findings (Song et al., 2017). In this earlier study, chalcones were reported to be potent Nrf2 activators, and, as expected, G. inflata, which contained abundant chalcones, showed significantly stronger activities than the other two species (Song et al., 2017). The content ratio of flavanone glycosides $(5,6) /$ chalcone glycosides (1) was significantly higher in G. uralensis, which was also consistent with previous report (Simmler et al., 2015). Revealing the differences among the chemical compositions of these three Glycyrrhiza species has been the subject of several studies. Kondo et al. (2007) determined nine major compounds and found that glycycoumarin, glabridin, and licochalcone A were specific markers for G. uralensis, G. glabra, and G. inflata, 
Table 3. Contents ( $\mu \mathrm{g} / \mathrm{g}$ ) of six flavonoids in G. uralensis, G. inflate, and G. glabra root samples.

\begin{tabular}{|c|c|c|c|c|c|c|c|c|}
\hline \multirow{2}{*}{ No } & \multirow{2}{*}{ Species } & \multirow{2}{*}{ Produing areas } & \multicolumn{6}{|c|}{ Contents $(\mu \mathrm{g} / \mathrm{g})$} \\
\hline & & & licochalcone A & liquiritigenin & glabridin & isoliquiritigenin & liquiritoside & isoliquiritoside \\
\hline 1 & G. inflata & Xinjiang & $7.74 \pm 0.12$ & $6.97 \pm 0.05$ & $13.51 \pm 0.22$ & $5.61 \pm 0.13$ & $10.93 \pm 0.29$ & $1.71 \pm 0.18$ \\
\hline 2 & G.uralensis & Neimenggu & $3.42 \pm 0.08$ & $8.12 \pm 0.04$ & $13.54 \pm 0.06$ & $8.04 \pm 0.10$ & $14.22 \pm 0.69$ & $8.11 \pm 0.18$ \\
\hline 4 & G. glabra & Ningxia & $2.42 \pm 0.03$ & $7.81 \pm 0.45$ & $22.02 \pm 1.41$ & $6.43 \pm 0.92$ & $12.88 \pm 1.57$ & $7.54 \pm 0.81$ \\
\hline 5 & G.uralensis & Yunnan & $1.56 \pm 0.20$ & $8.45 \pm 0.23$ & $7.38 \pm 1.40$ & $1.97 \pm 0.05$ & $34.1 \pm 1.28$ & $11.28 \pm 0.64$ \\
\hline 8 & G.uralensis & Gansu & $4.82 \pm 0.04$ & $7.56 \pm 0.22$ & $13.65 \pm 0.56$ & $2.69 \pm 0.15$ & $15.52 \pm 0.42$ & $0.82 \pm 0.59$ \\
\hline 9 & G.uralensis & Gansu & $4.04 \pm 0.45$ & $10.78 \pm 0.53$ & $10.13 \pm 0.29$ & $2.12 \pm 0.02$ & $18.77 \pm 0.91$ & $11.19 \pm 0.97$ \\
\hline 10 & G inflata & Neimenggu & $14.50 \pm 0.24$ & $9.32 \pm 0.31$ & $9.59 \pm 0.82$ & $8.39 \pm 0.31$ & $11.40 \pm 0.36$ & $3.55 \pm 0.62$ \\
\hline
\end{tabular}

Results expressed as mean \pm standard deviations of three measurements.

respectively. The influence of growing area condition was much less pronounced than that of species variation. It also can be seen that $G$. uralensis, the most important species of licorice, is distributed in different provinces, including Gansu, Neimenggu, Sichuan, and Yunnan through our detection. As whole, the new rapid quantitative method is suitable for the evaluation of the quality of raw licorice material.

In this investigation, we developed a new rapid analytical method for simultaneous determination of flavonoids in medicinal licorice. The detection system $\mathrm{UPC}^{2} / \mathrm{PDA}$ provided environmentally friendly separations by reducing the usage of toxic solvents, was more sensitive, and required less analysis time compared with HPLC/DAD analytical methods for the determination of flavonoids in licorice (Yang et al., 2016). The present $\mathrm{UPC}^{2}$ method can also reduce the material and solvent resources compared with ultra-fast liquid chromatography (UFLC) (Song \& Xu, 2013) and UHPLC/TQ-MS method (Zhu et al., 2016). Moreover, it has better detection sensitivity than high-performance thin-layer chromatography (HPTLC) detection (Arruda et al., 2017). In addition to the better sensitivity achieved, the validation of the new method confirmed its good linearity, accuracy, and precision. Furthermore, the method is suitable for the analysis of licorice from different origins.

\section{Acknowledgements}

This work was supported by National Natural Science Foundation of China (31501402).

\section{References}

Arruda, D., Valeria, M. W., Roy, U., Judith, N., Gunther, H., \& Eike, R. (2017). Qualitative and quantitative characterization of two licorice root species Glycyrrhiza glabra L. and Glycyrrhiza uralensis Fisch. by HPTLC, validated by HPLC and DNA sequencing. Journal of Planar Chromatography - Modern TLC, 30(6), 467-473. https://doi. org/10.1556/1006.2017.30.6.2.

Chen, C., Lu, J., Li, X., Zhou, L., Xie, L., Li, X., \& Song, F. (2017). Inheritance analysis and discovery of chloroplast paternal inheritance in interspecific crossing of Glycyrrhiza. Guihaia, 37, 162-168.

Commission Chinese. (2015). Pharmacopoeia of the people's republic of China. Beijing: China Medical Science Press.
Gao, X. J., Zhao, D., Zhao, J. J., Zhang, X., Wang, Y. H., \& Wang, H. Q. (2017). Herbal textural research on glycyrrhizae radix et rhizoma. Chin J Exp Tradit Med Form, 23(2), 193-198. Retrieved from: http:// en.cnki.com.cn/Article_en/CJFDTotal-ZSFX201702034.htm

Gong, X., Qi, N., Wang, X., Lin, L., \& Li, J. (2014). Ultra-performance convergence chromatography (UPC(2)) method for the analysis of biogenic amines in fermented foods. Food Chemistry, 162, 172-175. https://doi.org/10.1016/j.foodchem.2014.04.063. PMid:24874373.

ICH Expert Working Group. (1996). ICH harmonised tripartite guideline - Validation of analytical procedures text and methodology: Q2(R1). In International Conference on Harmonisation of Technical Requirements for Registration of Pharmaceuticals for Human Use (pp. 70-73). Geneva: The European Agency for the Evaluation of Medicinal Products. Retrieved from: https://www.ich.org/fileadmin/ Public_Web_Site/ICH_Products/Guidelines/Quality/Q2_R1/Step4/ Q2_R1_Guideline.pdf

Kondo, K., Shiba, M., Nakamura, R., Morota, T., \& Shoyama, Y. (2007). Constituent properties of licorices derived from Glycyrrhiza uralensis, G. glabra, or G. inflata identified by genetic information. Biological \& Pharmaceutical Bulletin, 30(7), 1271-1277. PMid:17603166.

Lee, C. K., Park, K. K., Lim, S. S., Park, J. H., \& Chung, W. Y. (2007). Effects of the licorice extract against tumor growth and cisplatininduced toxicity in a mouse xenograft model of colon cancer. Biological \& Pharmaceutical Bulletin, 30(11), 2191-2195. PMid:17978499.

Li, L., Xu, L., Peng, Y., He, Z., Shi, R., \& Xiao, P. (2012). Simultaneous determination of five phenylethanoid glycosides in small-leaved Kudingcha from the Ligustrum genus by UPLC/PDA. Food Chemistry, 131(4), 1583-1588. https://doi.org/10.1016/j.foodchem.2011.10.018.

Ministry of Health, Labour and Welfare. (2011). The japanese pharmacopoeia (6th ed.). Tokyo: Pharmaceuticals and Medical Devices Agency.

Qi, N., Gong, X., Feng, C., Wang, X., Xu, Y., \& Lin, L. (2016). Simultaneous analysis of eight vitamin $\mathrm{E}$ isomers in Moringa oleifera Lam. leaves by ultra performance convergence chromatography. Food Chemistry, 207, 157-161. https://doi.org/10.1016/j.foodchem.2016.03.089. PMid:27080892.

Simmler, C., Anderson, J. R., Gauthier, L., Lankin, D. C., McAlpine, J. B., Chen, S. N., \& Pauli, G. F. (2015). Metabolite Profiling and Classification of DNA-Authenticated Licorice Botanicals. Journal of Natural Products, 78(8), 2007-2022. https://doi.org/10.1021/acs. jnatprod.5b00342. PMid:26244884.

Simmler, C., Pauli, G. F., \& Chen, S. N. (2013). Phytochemistry and biological properties of glabridin. Fitoterapia, 90, 160-184. https:// doi.org/10.1016/j.fitote.2013.07.003. PMid:23850540. 
Song, E. Q., \& Xu, L. (2013). Fast chromatographic fingerprint analysis of Glycyrrhizae radix by ultra fast liquid chromatography. Journal of Liquid Chromatography \& Related Technologies, 36(19), 2749-2750. https://doi.org/10.1080/10826076.2012.725694

Song, W., Qiao, X., Chen, K., Wang, Y., Ji, S., Feng, J., Li, K., Lin, Y., \& Ye, M. (2017). Biosynthesis-based quantitative analysis of 151 secondary metabolites of licorice to differentiate medicinal glycyrrhiza species and their hybrids. Analytical Chemistry, 89(5), 3146-3153. https:// doi.org/10.1021/acs.analchem.6b04919. PMid:28192986.

Wang, Y. C., \& Yang, Y. S. (2007). Simultaneous quantification of flavonoids and triterpenoids in licorice using HPLC. Journal of Chromatography. B, Analytical Technologies in the Biomedical and Life Sciences, 850(1-2), 392-399. https://doi.org/10.1016/j. jchromb.2006.12.032. PMid:17224312.

Yang, R., Yuan, B., Ma, Y., Zhou, S., Zhang, H., Liu, J., Li, W., \& Liu, Y. (2016). Simultaneous determination of liquiritin,isoliquiritin,li quiritigenin and isoliquiritigenin in Glycyrrhiza uralensis Fisch., Glycyrrhiza glabra L., and Glycyrrhiza inflata Bat. by HPLC. Yaowu Fenxi Zazhi, 36, 1729-1736. https://doi.org/10.1021/acs.jafc.6b02954.
Yokota, T., Nishio, H., Kubota, Y., \& Mizoguchi, M. (1998). The inhibitory effect of glabridin from licorice extracts on melanogenesis and inflammation. Pigment Cell Research, 11(6), 355-361. https://doi. org/10.1111/j.1600-0749.1998.tb00494.x. PMid:9870547.

Zhang, Y. (2016). Study on glycyrrhizin in licorice $18 \alpha$ - and $18 \beta$-licorice acid content from different areas of China by HPLC. Pharmaceutical Biotechnology, 23, 55-57.

Zhou, Q., Gao, B., Zhang, X., Xu, Y., Shi, H., \& Yu, L. L. (2014). Chemical profiling of triacylglycerols and diacylglycerols in cow milk fat by ultra-performance convergence chromatography combined with a quadrupole time-of-flight mass spectrometry. Food Chemistry, 143, 199-204. https://doi.org/10.1016/j.foodchem.2013.07.114. PMid:24054231.

Zhu, Z. H., Tao, W. W., Li, J. P., Guo, S., Qian, D. W., Shang, E. X., Su, S. L., \& Duan, J. A. (2016). Rapid determination of flavonoids in licorice and comparison of three licorice species. Journal of Separation Science, 39(3), 473-482. https://doi.org/10.1002/jssc.201500685. PMid:26608595. 